\title{
MEMS gravity sensors for imaging density anomalies
}

\section{Andreas Noack, Richard Middlemiss, Abhinav Prasad, Giles Hammond}

Andreas Noack, Richard Middlemiss, Abhinav Prasad, Giles Hammond, "MEMS gravity sensors for imaging density anomalies," Proc. SPIE 10723, Optical Trapping and Optical Micromanipulation XV, 107230J (7 September 2018); doi: 10.1117/12.2323969

Event: SPIE Nanoscience + Engineering, 2018, San Diego, California, United States 


\title{
MEMS gravity sensors for imaging density anomalies
}

\author{
Andreas Noack ${ }^{\mathrm{a}}$, Richard Middlemiss ${ }^{\mathrm{a}}$, Abhinav Prasad ${ }^{\mathrm{a}}$, and Giles Hammond ${ }^{\mathrm{a}}$ \\ anstitute of Gravitational Research, SUPA, School of Physics and Astronomy, University of \\ Glasgow, Glasgow, UK
}

\begin{abstract}
Gravimeters measure small changes in the local gravitational acceleration. They are applied for environmental monitoring, oil \& gas prospecting and defence \& security. Gravimeters used in these applications have a remarkable sensitivity but at a cost of being bulky and very expensive. Recently, a micro-electrical mechanical system (MEMS) gravimeter has been developed, which was cheap, had a comparable sensitivity to commercial gravimeters and maintained its stability over long timescales $\left(10^{-6} \mathrm{~Hz}\right)$. In this paper we discuss to replace the current shadow sensor readout with an on-chip interferometer. This new readout has a higher sensitivity so that the device can be more robust and reduces the system size. The design of this readout is discussed and the first experimental results are presented. The new readout improves the imaging capabilities of density anomalies of the device.
\end{abstract}

Keywords: MEMS, Gravimeter, Interferometer, ...

\section{INTRODUCTION}

Gravimeters are used to measure tiny variations in the local gravitational acceleration $g$ allowing them to detect things difficult or impossible to see by other means underground. This capability can result in high impact, real-world applications of gravimeters in several fields. For example, gravimeters have been used in the oil and gas exploration industry ${ }^{1,2}$, in the defence industry to detect underground voids and tunnels ${ }^{3,4}$, in archaeology for detecting ancient ruins and structures ${ }^{5}$, in the geophysical mapping of sub-surface voids and sinkholes ${ }^{6}$, and in volcanology to monitor the flow of magma during prior to events. ${ }^{7,8}$

Despite the immense potential of gravimeters in subterranean imaging, costly instrumentation, associated complexity, and large form-factor have limited their widespread adoption so far. For example, a commercially available, field-portable gravimeter with an exquisite sensitivity of $1 \mu \mathrm{Gal} / \sqrt{\mathrm{Hz}}$ (where $1 \mu \mathrm{Gal} \approx 10 \mathrm{~nm} / \mathrm{s}^{2}$ ) could cost $>\$ 100 \mathrm{k}$ and can weigh $\geq 6 \mathrm{~kg} .{ }^{9}$ Further, such gravimeters have generally only been used for point measurements, and are financially risky for unsupervised remote observations.

Recently, researchers have developed MEMS-scale accelerometers with sensitivities which are at comparable with commercial gravimeters. ${ }^{10,11}$ These MEMS accelerometers, however, have not yet demonstrated a long long-term stability which is important for gravimetry. More recently, a MEMS-based gravimeter with excellent long-term stability, which promises at least an order of magnitude reduction in cost, size, and weight, has been developed by the authors. ${ }^{12,13}$

While field-portable ${ }^{14}$, the reported MEMS gravimeter is size limited by the use of a shadow sensor readout ${ }^{15}$ , and requires careful handling due to the presence of shock susceptible delicate MEMS-scale features because of the low resonance frequency of the device. To overcome these limitations, in this paper, the concept of a more robust and extremely sensitive interferometric readout based MEMS gravimeter is reported and the first experimental results are shown. An on-chip implementation of the interferometric readout and the use of microfabrication processes help achieving the sensor's small form factor. A more robust mechanical design coupled with the less than picometer displacement readout capability is expected to ensure a thermal noise limited sensitivity of $5 \mu \mathrm{Gal} / \sqrt{(\mathrm{Hz})}$.

Further author information: (Send correspondence to Giles Hammond)

Giles Hammond: E-mail: giles.hammond@glasgow.ac.uk, Telephone: +44 (0) 1413302258

Andreas Noack: E-mail: a.noack.1@research.gla.ac.uk, Telephone: +44 (0) 1413302258

Optical Trapping and Optical Micromanipulation XV, edited by Kishan Dholakia, Gabriel C. Spalding, Proc. of SPIE Vol. 10723, 107230J · C 2018 SPIE · CCC code: 0277-786X/18/\$18 · doi: 10.1117/12.2323969 


\section{DESIGN}

MEMS Design The gravimeter consists of a proof-mass $\mathrm{m}$ suspended by micromechanical suspensions of an equivalent spring constant $\mathrm{k}$. When aligned with the local gravitational field, the device displaces by $\Delta x$ in the direction of the field. This relation is described by the following equation:

$$
\Delta g=(k / m) \Delta x=\omega_{0}^{2} \Delta x,
$$

where $\Delta g$ is the minimum resolvable change in $g$ and $\omega_{0}$ the fundamental resonance frequency. Hence, to improve the acceleration sensitivity a lower resonant frequency and/or higher displacement sensitivity is required. As low resonant frequency MEMS accelerometers are fragile due to the fact that their springs are very soft, the designer has to trade off resonant frequency and robustness with the displacement sensitivity of the readout. As the previous demonstrated MEMS gravimeter was limited by the sensitivity of its displacement sensor at around $\pm 4 \mathrm{~nm}$ the resonance frequency had to be very low. Hence, an anti-spring geometry with a resonant frequency of $2.5 \mathrm{~Hz}$ was designed to measure the earth tides. ${ }^{12}$ Due to its three $6 \mu \mathrm{m}$ wide curved flexures it was very delicate.

Interferometer Design In this work, we propose to replace the shadow sensor with an interferometer. By measuring the relative phase between two interfering light beams an interferometer can measure a relative displacement. In a typical Michelson interferometer structure an incident laser beam is split by a beam splitter, both the split beams travel towards two mirrors, are reflected and then overlapped at the same beam splitter again. When one mirror is fixed and the other is moving along the laser beam direction, the output of the interferometer $P$ is described by the following equation:

$$
P=P_{0} \cos (2 \pi \Delta L / \lambda),
$$

where $P_{0}$ is the input laser power, $\Delta L$ is the relative arm difference between the mirrors and the beam splitter and $\lambda$ the incident laser wavelength. When one of the mirrors is attached to the proof-mass and the other mirror is fixed to the frame a displacement of the proof mass relative to the frame can be measured.

In comparison to the shadow sensor readout, the interferometer has several advantages. First, the interferometer is built on the same chip using common fabrication steps as the gravimeter. This eases assembly and reduces complexity of the system. However, this shifts the difficulties to the fabrication as optical components like beam splitters and mirrors have to be built from the same substrate material as the gravimeter, which is silicon in our case. Second, while the miniaturization of the shadow sensors is quite challenging, the proposed device is expected to be much smaller. Third, an interferometer has a much higher sensitivity than the shadow sensor because of its high optical gain. Hence, the resonant frequency of the device can be increased for improving the device robustness while still achieving superior sensitivity over the shadow sensor. And fourth, this the on-chip interferometer design can be easily integrated into any other MEMS device where an in-plane displacement of the MEMS device is to be measured with a high degree of precision.

A Michelson interferometer topology is preferred while designing the device as it provides twice the sensitivity compared to a Mach-Zehnder configuration. In addition, the beam path on the chip is simplified to reduce the number of reflections and also the optical losses. As the thermal noise floor of the previously reported MEMS gravimeter was estimated to be around $5 \mu \mathrm{Gal} / \sqrt{(\mathrm{Hz})}$, we designed a more robust gravimeter such that the measurements are limited by the thermal noise limit, assuming an easily achievable sensitivity of the interferometer $( \pm 0.5 \mathrm{pm})$. With these requirements a gravimeter with a resonant frequency of $16 \mathrm{~Hz}$ could be designed. To eliminate laser frequency noise coupling, which is expected to dominate at low frequencies, both arms of the interferometer have the same arm length when the gravimeter is placed in the local gravity field.

As the beam splitter is the central element in the Michelson topology a lot of effort went into its design. Two different beam splitter designs have been discussed in the extant literature: designs based on a thin beam splitters ${ }^{16}$ or a thick beam splitters. ${ }^{17}$ The thick beam splitter designs were developed to counteract the effect of a wavelength dependent transmission and reflection in thin beam splitters which was limiting in spectroscopy applications. The wavelength dependencies were caused by the beam splitter's two parallel walls forming a Fabry-Perot-cavity. However, in the thick beam splitters as one arm of the interferometer is buried in silicon this, first, causes wave dispersion, limiting the interferometer visibility. ${ }^{18}$ Second, it gives rise to temperature coupling to the arm length of the interferometer, deteriorating its sensitivity. Hence, we opt to use a thin beam 


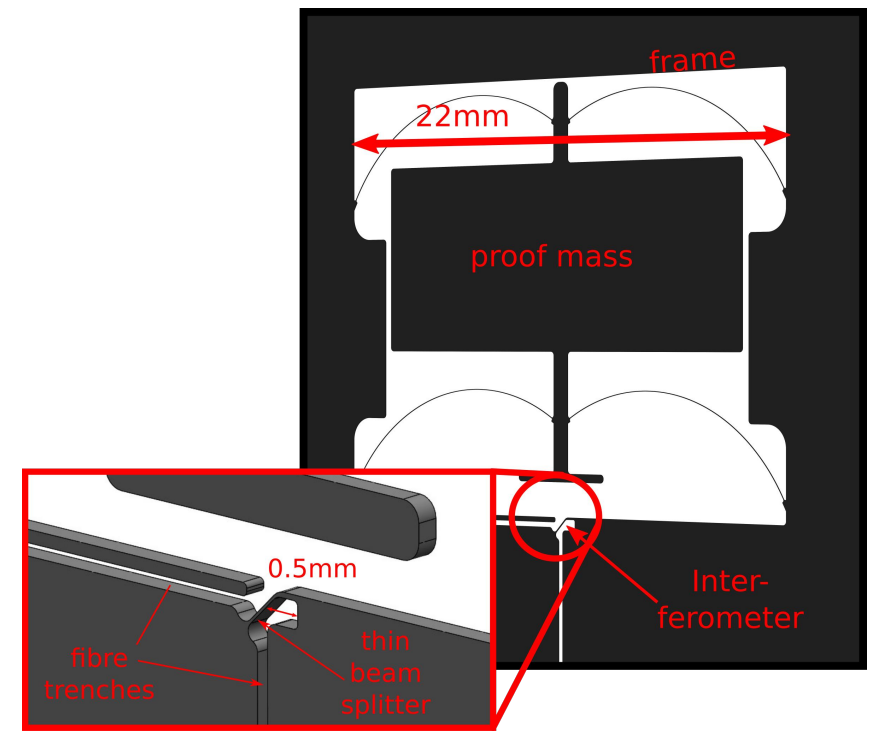

Figure 1. Top Right: Illustration of the complete MEMS Gravimeter and the interferometer. Only the beam splitter (close up in the bottom left) and the two fibre trenches for input and output fibre are shown.

splitter architecture in our design. The incident angle to the beam splitter is 55 deg to achieve a reflection and a transmission coefficient of 0.5 each (from the Fresnel equation).

Alternatively, using a grating instead of a beam splitter would allow for an all-reflective interferometer, which would not show any wave dispersion at all and with a reduced thermal coupling. ${ }^{19}$ However, a major design challenge was to find a good geometry to separate the incident, reflected, and output laser beams.

The light needs to be reflected and returned to the beam splitter. A simple silicon wall reflects roughly $30 \%$ of the incident light at $0 \mathrm{deg}$ incident angle. If further improvements to the interferometer performance is necessary these simple reflectors can be optimised by a metallisation step ${ }^{18}$ or bragg gratings. The latter are especially interesting as layers of air and silicon can be stacked to achieve high reflection. Due to the large difference in the refractive index only two to three stacks are required to achieve a reflectivity of roughly $99 \%$. However, both approaches increase the design and fabrication related complexity.

Although, interferometers can be run in open-loop configurations ${ }^{20}$, force feedback is often required to push the sensitivity down further. ${ }^{21}$ Hence, on-chip temperature control and electrostatic feedback will be used to hold the MEMS proof mass still. At first we will use a digital FPGA-based feedback control to stabilise the interferometer to the Mid-Fringe. Here, the working point of the interferometer is half way up the fringe. However, this readout having the highest optical gain is limited by the intensity noise of the laser. Hence a more sophisticated readout stabilising the interferometer to the dark-fringe would result in a higher sensitivity readout. After all, this readout will be limited by the shot noise of the laser. In our calculation a shot noise limited mid-fringe readout would have a sensitivity of a couple of femotmeter with a laser power of a few Milliwatt (calculations adapted from Heinzel ${ }^{22}$ ).

The complete device, the MEMS gravimeter interfaced to an interferometer (without the force-feedback actuator) can be seen in Fig. 1. For design simplicity reasons, the beam splitter incident angle is kept at $45 \mathrm{deg}$. Four flexures $(13 \mu \mathrm{m}$ thick) can be seen running from the frame to the proof mass. When the device is positioned in the local gravity field the proof mass is displaced. The interferometer is at the far bottom of the device. Light is coupled on the chip with a fibre from the left. The output light is coupled through a another fibre to a photo diode (not shown).

For initial testing a laser is assembled off chip and light is fed through fibres onto the chip. At the output port of the beam splitter the light is coupled to another fibre and directed towards a photo diode. Later the photo diode will be integrated on the same chip $^{23}$ as will the laser, both, manufactured at our own clean room. 


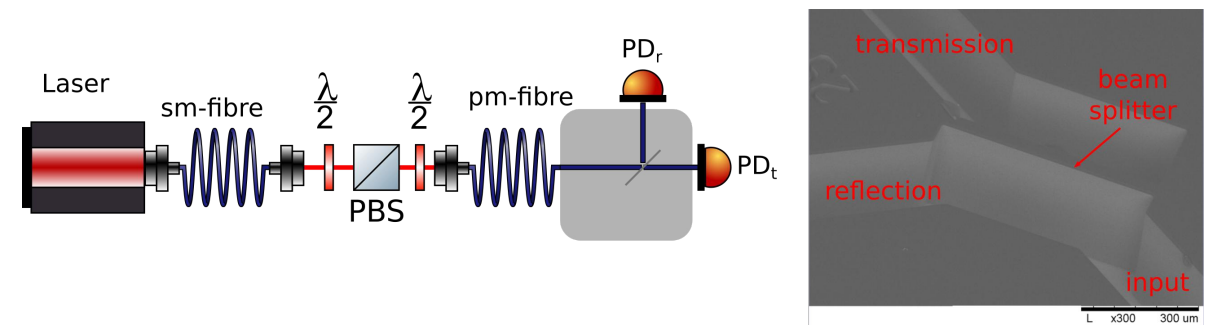

Figure 2. Setup for testing the beam splitter. sm: single mode; $\lambda / 2$ : lambda half-wave plate; PBS: polarisation beam splitter; pm: polarisation maintaining; PD: photo diode.

Fabrication As discussed earlier, the interferometer is built on the same silicon chip and using the same fabrication steps as the gravimeter. A detailed discussion on the fabrication process of the gravimeter was reported by Middlemiss et al. ${ }^{12}$. In short, a $240 \mu \mathrm{m}$ thick single crystal silicon wafer is used for the device fabrication. To etch and release the gravimeter, after coating the wafer with a positive photoresist, a "halo" mask design outlining the structure of the gravimeter is used in conjunction with the "Bosch process", a deep reactive ion etch (DRIE), to etch the device. We optimised the process to allow for a high aspect ratio etch which is necessary for the $13 \mu \mathrm{m}$ wide, $240 \mu \mathrm{m}$ deep flexures. The interferometer is now being etched into the silicon using the same technique. Its components are outlined with the same 'halo mask' as described above to guarantee the same etch quality. The sidewall smoothness and verticality of the etch are critical to achieve a good interferometer performance. While the former will determine how much light is scattered upon reflection, the latter is crucial as it determines the alignment of the interferometer and, therefore, how well the laser beams interfere. We have optimised the process to allow for a high aspect ratio etch which is necessary for the $13 \mathrm{um}$ wide, $220 \mathrm{um}$ deep flexures. We achieved a verticality of $90 \pm 0.1 \mathrm{deg}$ with scalloping of around $80-90 \mathrm{~nm}$. With an additional fabrication process these scallops can be reduced further. ${ }^{24}$

\section{EXPERIMENT}

Extensive characterization of the fabricated beam splitters is critical to the functioning of the designed on-chip interferometer. The effect of thickness and input polarisation on the output of the fabricated beam splitters are discussed.

The reflection of a thin beam splitter is described by: $:^{25}$

$$
\begin{aligned}
R_{\mathrm{BS}} & =\frac{2 R^{2}(1-\cos (\Delta \Phi))}{\left(1+R^{2}-2 R \cos (\Delta \Phi)\right.}, \text { with } \\
\Delta \Phi & =4 \pi f n_{\mathrm{si}} d \cos \left(\theta_{i}\right),
\end{aligned}
$$

where $R$ is the Fresnel reflection coefficient, $f$ the laser frequency, $n_{\mathrm{si}}$ the refractive index of silicon, $d$ the thickness of the beam splitter and $\theta_{i}$ the incident angle. Assuming zero losses the transmission $T$ can be calculated by $T=1-R$. From Eq. 3 the reflection changes periodically with the thickness of the beam splitter. Beam splitters with thicknesses (from 4 to $25 \mu \mathrm{m}$ ) where reflection and transmission were maximal were designed and tested.

According to the Fresnel reflection the throughput power also depends on the polarisation. The setup shown in Fig. 2 was used to measure the dependency of the input polarisation to the transmission and reflection. A standard fibre coupled Thorlabs $1550 \mathrm{~nm}$ laser was used as a laser source. The laser power through the polarisation beam splitter (PBS) was maximised using the first half-wave plate. The light was coupled to a polarisation maintaining fibre, while the other end was pointed towards the beam splitter under test. An SEM image of a beam splitter $(24.9 \mu \mathrm{m}$ thick $)$ is shown on the right hand side in Fig. 2. The reflected and the transmitted light beams were coupled into two large core single-mode fibres and the power was measured using two photo diodes. The second half-wave plate was turned to change the polarisation of the incident light to the beam splitter. Recording the power divided by the input power while stepwise changing the input polarisation resulted in the plot shown in Fig. 3. By rotating the half-wave plate respectively changing between s- and p-polarisation a periodic power increase and decrease was visible in reflection. The shape is exactly opposite 


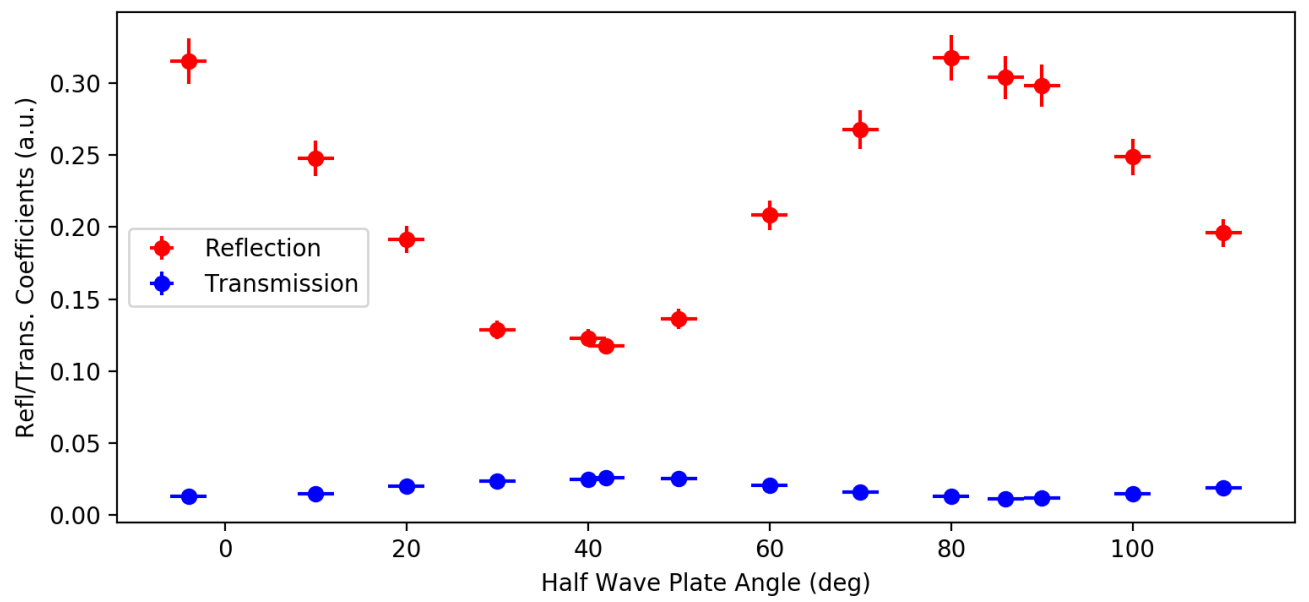

Figure 3. Measurement of the reflection and transmission coefficient when the second half-wave plate in Fig. 2 is rotated.

in the transmission confirming light splitting using the designed beam-splitters. However, the magnitude of the reflection and the transmission coefficient is lower than the expected value of 0.5 for both for complete s-polarised light. According to the Fresnel equations changing from s- to p- polarised light a change in the reflection coefficient from 0.5 to 0.2 is expected. In return the transmission should inversely change too. In Fig. 3 only a change from 0.32 to 0.13 for the reflection and a change of 0.1 to 0.03 for the transmission coefficient is observed. Also, both coefficients don't add up to 1. This means there are excesses losses in the current setup. These could be due to laser beam diffraction, imperfect fibre alignment, non-vertical beam splitter walls and optical losses due to scattering light. Also, the fabrication process hadn't been optimised yet so the beam splitter thickness was not exactly at the design thickness which could result in a decreased reflection.

\section{CONCLUSION}

Recently a MEMS Gravimeter has been designed with the potential of revolutionising the gravimetry survey market due to its high sensitivity, stability and cheapness. In this paper the potential improvements to the sensitivity and robustness to the MEMS gravimeter by using an on-chip interferometer have been discussed. Its design has been presented and first experimental results on the characterisation of a thin beam splitter design have been shown. These results demonstrate the potential use of this type of beam splitter whilst also emphasising that polarisation and frequency stability is crucial for long term stability. The MEMS Gravimeter will have a resonant frequency of $16 \mathrm{~Hz}$ to be more robust than the previous version. With a pessimistic assesment of the interferometer's displacement sensitivity (of around $1 \mathrm{pm}$ ), it is likely that the interferometer will not be the limiting noise source of the gravimeter; instead, a thermal noise floor of $5 \mu \mathrm{Gal} / \sqrt{(\mathrm{Hz})}$ will be the limit of the device sensitivity. Work is now underway to integrate the complete interferometer with the gravimeter to evaluate its performance and stability.

\section{REFERENCES}

[1] Barnes, G. and Barraud, J., "Imaging geologic surfaces by inverting gravity gradient data with depth horizons," GEOPHYSICS 77(1), G1-G11 (2012).

[2] Rim, B. and Li, Y., "Borehole vector gravity: A numerical study," (09 2013).

[3] Butler, D. K., "Microgravimetric and gravity gradient techniques for detection of subsurface cavities," GEOPHYSICS 49(7), 1084-1096 (1984). 
[4] Romaides, A. J., Battis, J. C., Sands, R. W., Zorn, A., Jr, D. O. B., and DiFrancesco, D. J., "A comparison of gravimetric techniques for measuring subsurface void signals," Journal of Physics D: Applied Physics 34(3), 433 (2001).

[5] Pnisov, J. and Pateka, R., "The use of microgravity technique in archaeology: A case study from the st. nicolas church in pukanec, slovakia," Contributions to Geophysics and Geodesy 39(3) (2009).

[6] Kaufmann, G., "Geophysical mapping of solution and collapse sinkholes," Journal of Applied Geophysics 111, $271-288$ (2014).

[7] Battaglia, M., Gottsmann, J., Carbone, D., and Fernndez, J., "4d volcano gravimetry4d volcano gravimetry," Geophysics 73(6), WA3 (2008).

[8] Carbone, D., Poland, M. P., Diament, M., and Greco, F., "The added value of time-variable microgravimetry to the understanding of how volcanoes work," Earth-Science Reviews 169, 146 - 179 (2017).

[9] Michel, V. C., P., W. S. D., and Olivier, F., "Uncertainty of absolute gravity measurements," Journal of Geophysical Research: Solid Earth 110(B5).

[10] Krishnamoorthy, U., Olsson, R., Bogart, G., Baker, M., Carr, D., Swiler, T., and Clews, P., "In-plane mems-based nano-g accelerometer with sub-wavelength optical resonant sensor," Sensors and Actuators A: Physical 145-146, 283 - 290 (2008). Special Issue: Transducers/07 Eurosensors XXI, The 14th International Conference on Solid State Sensors, Actuators and Microsystems and the 21st European Conference on SolidState Transducers.

[11] Pike, W., Calcutt, S., Standley, I., Mukherjee, A. G., Temple, J., Warren, T., Charalambous, C., Liu, H., Stott, A., and McClean, J., "A silicon seismic package (ssp) for planetary geophysics," (03 2016).

[12] Middlemiss, R. P., Samarelli, A., Paul, D. J., Hough, J., Rowan, S., and Hammond, G. D., "Measurement of the earth tides with a mems gravimeter," Nature 531, 614-617 (mar 2016).

[13] Middlemiss, R. P., Bramsiepe, S. G., Douglas, R., Hild, S., Hough, J., Paul, D. J., Samarelli, A., Rowan, S., and Hammond, G. D., "Microelectromechanical system gravimeters as a new tool for gravity imaging," Philosophical Transactions of the Royal Society of London A: Mathematical, Physical and Engineering Sciences 376(2120) (2018).

[14] Middlemiss, R. P., Bramsiepe, S. G., Douglas, R., Hough, J., Paul, D. J., Rowan, S., and Hammond, G. D., "Field tests of a portable mems gravimeter.," Sensors (Basel, Switzerland) 17 (Nov 2017).

[15] Bramsiepe, S. G., Loomes, D., Middlemiss, R. P., Paul, D. J., and Hammond, G. D., "A high stability optical shadow sensor with applications for precision accelerometers," IEEE Sensors Journal 18 (May 2018).

[16] Saadany, B., Bourouina, T., Malak, M., Kubota, M., Mita, Y., and Khalil, D., "A miniature michelson interferometer using vertical bragg mirrors on soi," in [IEEE/LEOS International Conference on Optical MEMS and Their Applications Conference, 2006.], 50-51 (Aug 2006).

[17] Saadany, B., Omran, H., Medhat, M., Marty, F., Khalil, D., and Bourouina, T., "Mems tunable michelson interferometer with robust beam splitting architecture," in [2009 IEEE/LEOS International Conference on Optical MEMS and Nanophotonics], 49-50 (Aug 2009).

[18] Warashina, Y., Suzuki, T., Kasamori, K., Okumura, R., Matsuo, Y., and Takemura, M., "Mems based miniature ft-ir engine with built-in photodetector," (2014).

[19] Sun, K.-X. and Byer, R. L., "All-reflective michelson, sagnac, and fabry-perot interferometers based on grating beam splitters," Opt. Lett. 23, 567-569 (Apr 1998).

[20] Dandridge, A., Tveten, A. B., and Giallorenzi, T. G., "Homodyne demodulation scheme for fiber optic sensors using phase generated carrier," IEEE Transactions on Microwave Theory and Techniques 30, 16351641 (Oct 1982).

[21] Hild, S., Grote, H., Degallaix, J., Chelkowski, S., Danzmann, K., Freise, A., Hewitson, M., Hough, J., Lck, H., Prijatelj, M., Strain, K. A., Smith, J. R., and Willke, B., "DC-readout of a signal-recycled gravitational wave detector," Classical and Quantum Gravity 26, 055012 (feb 2009).

[22] Heinzel, G., Advanced optical techniques for laser-interferometric gravitational-wave detectors, $\mathrm{PhD}$ thesis, University of Hannover (1999).

[23] Ghisetti, E. A., Dumas, D., Kirdoda, J., Gallacher, K., Millar, R. W., Mirza, M. M. A., and Paul, D. J., "Germanium on silicon single-photon avalanche detectors using silicon-on-insulator substrates (conference presentation)," (2018). 
[24] Defforge, T., Song, X., Gautier, G., Tillocher, T., Dussart, R., Kouassi, S., and Tran-Van, F., "Scalloping removal on drie via using low concentrated alkaline solutions at low temperature," Sensors and Actuators A: Physical 170(1), $114-120$ (2011).

[25] Homes, C. C., Carr, G. L., Lobo, R. P. S. M., LaVeigne, J. D., and Tanner, D. B., "Silicon beam splitter for far-infrared and terahertz spectroscopy," Appl. Opt. 46, 7884-7888 (Nov 2007). 